\title{
The properties of SIRT, TVM, and DART for 3D imaging of tubular domains in nanocomposite thin-films and sections
}

\author{
Delei Chen $^{\mathrm{a}, \mathrm{b}}$, Bart Goris ${ }^{\mathrm{c}}$, Folkert Bleichrodt ${ }^{\mathrm{d}}$, Hamed Heidari Mezerji ${ }^{\mathrm{c}}$, Sara Bals ${ }^{\mathrm{c}}$, \\ Kees Joost Batenburg ${ }^{\mathrm{d}}$, Gijsbertus de With ${ }^{\mathrm{a}}$, Heiner Friedrich ${ }^{\mathrm{a}, *}$ \\ a Laboratory of Materials and Interface Chemistry, Department of Chemical Engineering and Chemistry, Eindhoven University of Technology, Den Dolech 2, \\ 5612 AZ Eindhoven, The Netherlands \\ ${ }^{\mathrm{b}}$ Dutch Polymer Institute (DPI), P.O. Box 902, 5600 AX Eindhoven, The Netherlands \\ c EMAT, University of Antwerp, Groenenborgerlaan 171, B-2020 Antwerp, Belgium \\ d Centrum Wiskunde \& Informatica, Science Park 123, NL-1098XG Amsterdam, The Netherlands
}

\section{A R T I C L E I N F O}

Article history:

Received 22 April 2014

Received in revised form 25 July 2014

Accepted 3 August 2014

Available online 19 August 2014

Keywords:

Electron tomography

Reconstruction algorithm

Simultaneous iterative reconstruction

technique (SIRT)

Total variation minimization (TVM)

Discrete algebraic reconstruction (DART)

Beam-sensitive material

\begin{abstract}
A B S T R A C T
In electron tomography, the fidelity of the 3D reconstruction strongly depends on the employed reconstruction algorithm. In this paper, the properties of SIRT, TVM and DART reconstructions are studied with respect to having only a limited number of electrons available for imaging and applying different angular sampling schemes. A well-defined realistic model is generated, which consists of tubular domains within a matrix having slab-geometry. Subsequently, the electron tomography workflow is simulated from calculated tilt-series over experimental effects to reconstruction. In comparison with the model, the fidelity of each reconstruction method is evaluated qualitatively and quantitatively based on global and local edge profiles and resolvable distance between particles. Results show that the performance of all reconstruction methods declines with the total electron dose. Overall, SIRT algorithm is the most stable method and insensitive to changes in angular sampling. TVM algorithm yields significantly sharper edges in the reconstruction, but the edge positions are strongly influenced by the tilt scheme and the tubular objects become thinned. The DART algorithm markedly suppresses the elongation artifacts along the beam direction and moreover segments the reconstruction which can be considered a significant advantage for quantification. Finally, no advantage of TVM and DART to deal better with fewer projections was observed.
\end{abstract}

(c) 2014 Elsevier B.V. All rights reserved.

\section{Introduction}

Electron tomography (ET) has nowadays become a standard tool for materials research to reveal the three dimensional (3D) morphology of specimens with nanometer resolution [1-4]. Knowledge of a materials 3D morphology is critical to understanding the material properties, such as, e.g., the efficiency of polymer photovoltaic cells [5,6]. ET consists of three basic steps [2]. First, a series of 2D projections from multiple directions of the object of interest is acquired in a transmission electron microscope (TEM). This is conventionally done by tilting the object over a large angular range at small tilt increments. Second, the series of projections are aligned with respect to a common origin and tilt axis, correcting for unavoidable displacements during data acquisition. Third, the electron tomogram (3D intensity map) of the object is reconstructed from the tilt series by numerical algorithms.

\footnotetext{
* Corresponding author. Tel.: +31 402473041.

E-mail address: h.friedrich@tue.nl (H. Friedrich).
}

ET is mainly used for qualitative studies where a 3D visualization of different nanostructures is required. Nevertheless a strong trend towards obtaining 3D quantitative information from electron tomograms is ongoing that is currently often hampered by the low reconstruction quality [7]. Several challenges impede obtaining high-quality reconstructions [8-10]. The most significant challenge is that the tilt range for ET is limited (often $< \pm 80^{\circ}$ ) because of the sample, the sample holder or the microscope stage [11]. As a consequence, the limited tilt range leads to a missing angular range of information referred to as "missing wedge" [2,12]. The reconstruction quality is significantly affected by the missing wedge, i.e. elongation in the reconstruction along the beam direction [13]. Another key challenge is the low contrast and low signal-to-noise ratio (SNR) that can be obtained in the projection images, especially for beam sensitive materials $[2,8,14]$. The reason of low contrast is that, e.g., polymers and composites thereof, mainly consist of light elements with small differences in density or composition. In addition, polymers are often very sensitive to electron irradiation and will be shrunk or even bubble during data acquisition if one goes beyond a tolerable cumulative 
electron dose [15-17]. Therefore, in order to avoid radiation damage and to preserve the structure of the object, only a limited number of electrons can be used throughout data acquisition, thus, leading to low SNR in the projections of the tilt-series.

To overcome the aforementioned challenges, various methods have been proposed in the literature $[1,3,8]$. Instead of a single tilt axis, dual tilt axis has been used during acquisition, thereby reducing the missing wedge to a "missing pyramid" $[18,19]$. Nevertheless, the problem of missing information still persists. The ideal tilt range $\pm 90^{\circ}$ can be reached by fabricating the material of interest into a needle shaped specimen. By mounting the sample in a special specimen holder, an angular tilt range of 360 degrees can be achieved thus completely removing the missing wedge $[20,21]$. However, fabrication of a needle-shaped specimen requires materials that are mechanically stable enough which hampers the application to many polymer composites. Finally, to enhance the reconstruction quality, different angular sampling procedures and reconstruction schemes have been suggested and implemented [22-24].

Commonly used reconstruction methods are the weighted backprojection method (WBP) and iterative methods such as simultaneous iterative reconstruction technique (SIRT) [13,2527]. The WBP method has been one of the most widely used algorithms [13,25]. Apart from high computational efficiency, the major advantage of WBP is that the outcome of the reconstruction is thoroughly determined by the experimental data as all steps in the algorithm are linear $[13,28]$. However, the main disadvantage of WBP is that the reconstruction quality is sensitive to the limited tilt range [28]. In contrast, the SIRT method generates reconstructions yielding good visual quality from fewer projections and even from noisy data $[26,29]$. Therefore, SIRT gradually becomes an increasingly popular reconstruction method used in electron tomography, although it is computationally more expensive. Recently, more advanced reconstruction algorithms have been proposed, such as the discrete algebraic reconstruction technique (DART) [22] and the total variation minimization based reconstruction technique (TVM) [23]. Using prior knowledge of the specimen such as that the specimen contains only a limited number of phases, i.e., a discrete number of gray levels, aids in solving the ill-posed inversion (reconstruction) problem. For example, the DART algorithm actually segments (binarizes) the reconstruction during the iterative process. DART reconstructions are directly quantifiable and have been successfully applied for the 3D characterization of catalytic $\mathrm{CuO}$ nanoparticles and zeolite materials $[30,31]$. The TVM method is developed based on compressed sensing. It incorporates the prior knowledge that the boundary of the specimen is sparse in the reconstruction [23]. Using this algorithm, the elongation artifacts and noise in the reconstruction are reduced and the sharpness of the edges is significantly improved. The advantages of the TVM method have demonstrated by the 3D reconstruction of $\mathrm{FeO}$ nanoparticles [32] and $\mathrm{PbSe} / \mathrm{CdSe}$ core-shell nanoparticles [23].

The performance of above advanced reconstruction algorithms have been mainly studied based on experimental data [22,33] which generally lack the ground truth of the specimens. Nevertheless, some studies have incorporated known phantoms or models into the performance comparison, but these models were based on data that are obtained with a very high SNR in the projections $[32,34,35]$. Hence, no study has included effects of limited electron dose, which we consider the most crucial physical limit to 3D imaging of beam sensitive materials. Thus for beam sensitive materials, detailed studies on the performance of reconstruction algorithms in combination with limited electron dose and varying tilt schemes are lacking.

The aim of this work is therefore to fill this gap by evaluating the fidelity of SIRT, TVM, and DART reconstructions in dependence of a limited total electron dose and a variety of possible acquisition schemes. A comparison to WBP will be presented as a reference. As model structure we focus on the large range of functional nanocomposites composed of tubular domains in a matrix with slab-geometry, i.e. thin-films or thin-sections. Our approach is built on simulating the entire workflow of the bright-field ET from projections, over experimental imaging and recording effects to 3D reconstructions, finalized by a qualitative and quantitative comparison of the initial model and the reconstruction. The reconstruction fidelity is first assessed by image quality. Subsequently, local and global edge profiles and edge spread functions are employed to quantify the resolution in the reconstructions. Moreover, we evaluate the resolvable separation between particles (connectivity and percolation) which is a key question for many functional composites such as photovoltaic bulk heterojunctions or conductive CNT/polymer nanocomposites.

\section{Materials and methods}

In this section, we first briefly introduce SIRT, TVM and DART, and then present the simulation approach and evaluation methods [36].

\subsection{Reconstruction methods}

In iterative methods, the reconstructed object is assumed as an unknown array $\boldsymbol{x}$. According to the measured projections $\boldsymbol{b}$, a set of algebraic equations can be formulated $\boldsymbol{A x}=\boldsymbol{b}$, where $\boldsymbol{A}$ is a weighting matrix $[23,25,26]$. The elements of the weighting matrix $\boldsymbol{A}$ represent the contribution of a specific voxel to each projection ray. They can be obtained using different interpolation schemes and thus may yield slight differences in the final reconstruction [37-39]. In order to obtain a reconstruction of the object, $\boldsymbol{x}$ has to be extracted from the projection equation based on a limited data set.

\subsubsection{SIRT}

The SIRT algorithm is an iterative method based on the algebraic reconstruction technique $[25,26,29]$. The algorithm starts from an initial reconstruction, which is usually obtained by simply assuming a uniform data array or by a simple back-projection from the tilt series. The reconstruction is re-projected along the same tilt angles as the angles used to acquire projections during the experiment, referred as forward projection. The error between the re-projections and the measured projections are calculated and is referred to as "projection error", and then simultaneously backprojected to refine the reconstruction. This process is iterated until a stop criterion is reached, conventionally the number of iterations. Using this method with few iterations, the noise in the reconstruction can be suppressed, yielding good looking reconstructions.

\subsubsection{TVM}

As mentioned above, TVM in the used implementation is an iterative method based on compressive sensing $[23,33]$. It assumes that the specimen has only a few components and that the gradient of the specimen is sparse. Based on this sparsity assumption, the aim of TVM is to find the reconstructed object which has a minimum norm of the discrete gradient, i.e. the total variation. This method is implemented by simultaneously minimizing the projection error and the total variation of the reconstructed object, and can be represented by

$\boldsymbol{x}=\operatorname{argmin}_{\boldsymbol{x}}\left[T V(\boldsymbol{x})+\frac{\mu}{2} \boldsymbol{A} \boldsymbol{x}-\boldsymbol{b}^{2}\right]$ 
where the $\operatorname{TV}(\boldsymbol{x})$ is the total variation of the reconstructed object $\boldsymbol{x}$, $\mu$ is a regularization parameter, and $\left\|{ }^{\circ}\right\|^{2}$ denotes a sum of squared difference. The projection error is represented by $\| \boldsymbol{A x}$ - $\boldsymbol{b} \|$. As can be seen from this equation, the regularization parameter $\mu$ is very important. A large value of regularization parameter $\mu$ will result in a quasi-SIRT reconstruction, in which artifacts from noise and the missing wedge may be preserved. A small value of $\mu$ will reduce noise in the reconstruction, but small features in the object will also be removed. Therefore, great care should be taken when choosing the value of the regularization parameter. Although the ground truth is known in the simulation experiments performed in this work, this knowledge is not incorporated when estimating a correct value for the penalty parameter yielding a more realistic workflow.

\subsubsection{DART}

DART is an iterative algorithm that was developed for objects which consist of only few materials, corresponding to a few distinct gray levels in the reconstruction $[22,40]$. This prior knowledge is combined with a continuous iterative reconstruction algorithm (such as SIRT) by introducing a discretization step. The algorithm starts from a SIRT reconstruction to obtain information of gray levels in the reconstructed object. A threshold is then selected and applied to segment the reconstruction. Boundary pixels are detected from the segmented reconstruction, which are all pixels that have at least one neighboring pixel in a different segmentation class. The remaining pixels are referred as nonboundary pixels, and they are assigned to the gray levels that correspond to either background or objects. Afterwards, the SIRT method is employed again, but only boundary pixels are updated in each iteration step. The non-boundary pixels remain fixed throughout this process. In this way, the number of unknown pixels is extremely reduced. The update of the boundary is repeated until the method converges. In the end, a reconstruction is obtained, which is segmented and can be used for further quantification of structural properties.

\subsection{Simulation approach and evaluation methods}

In this section, we will introduce our simulation approach and the evaluation methods [36]. As shown in Fig. 1, the entire simulation contains of four steps: model definition, image formation and recording, reconstruction, and quantitative comparison.

\subsubsection{Model definition}

Our model approximates the structures of a large range of functional nanocomposites, such as a bulk heterojunction composed of P3HT nanowires in a PCBM matrix[6,41] or carbon nanotubes in a polymer matrix [42].The ingredients of the model system therefore consist of a polymer matrix (background) having a slab-geometry and rod-like nanofillers (object), as can be seen in Figs. 1 and S1. The rod-like filler, such as P3HT nanowires and carbon nanotubes, is simplified to a cylinder with spherical end caps which has a $200 \mathrm{~nm}$ length and $20 \mathrm{~nm}$ diameter. The thickness of the matrix is $200 \mathrm{~nm}$ as conventionally prepared by spin coating (bulk heterojunction) [6] or by (Cryo-)ultramicrotomy (CNT composite) [43]. In order to bridge the realms of medium resolution cryo-electron tomography to high resolution 3D imaging, the sampling in the model is set to $1 \mathrm{~nm} /$ pixel, which we used often in our experimental work.

\subsubsection{Image formation}

Our image formation model includes three main steps in electron microscopy: electron-specimen interaction, the optical system, and the recording process [44-46].

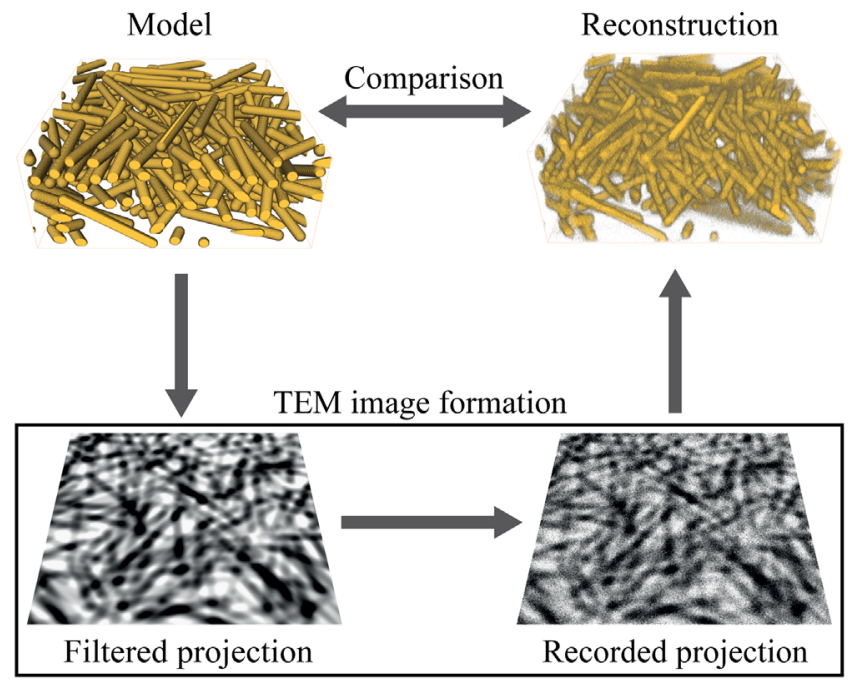

Fig. 1. Flowchart of the simulation approach. First, a well-defined model system is generated, which consists of a polymer matrix and rod-like nanofillers. Second, TEM images of the model are calculated based on mass-thickness contrast with a low-pass filter and noise. After calculating the tilt series, reconstruction is carried out. In the end, qualitative and quantitative comparisons of the reconstruction to the model are implemented to investigate the performance of reconstruction algorithms.

As an incident beam $I_{0}$ passes through a specimen with thickness $t$, the number of transmitted electrons owing to electron-specimen interaction decreases exponentially as a function of object thickness: $I_{\mathrm{t}}=I_{0} \exp \left(-t / \Lambda_{\mathrm{t}}\right)$, where $\Lambda_{\mathrm{t}}$ is the meanfree-path (MFP) length of the specimen $[46,47]$. The ideal projection formed by the transmitted electrons is transferred by the electron optical system of the TEM into the detector plane. In cooperation with aberration of the objective and defocus used for imaging, the optical system is modeled as a low-pass filter in first approximation. The transferred projection is thus simulated by the ideal projection with this low-pass filter, referred as filtered projection. Finally, the filtered projection is recorded by the TEM electron detector, i.e., a charge-coupled device (CCD) camera. The recording process introduces shot noise and CCD noise [48-50]. The intensity of the final projection is calculated by adding both shot noise and CCD noise to the filtered projection. More details can be found in the Supplementary information (SI), Section 1.2.

\subsubsection{Acquisition parameters and reconstruction}

In order to assess the ability of reconstruction methods dealing with noise, especially for beam sensitive polymer composites, we employ three different total electron doses $I_{\text {total }}$, which roughly correspond to electron doses commonly employed for cryo-ET of vitrified specimens in their native liquid condition (highly beam sensitive, $10^{2} \mathrm{e} / \AA^{2}$ ), for polymers and composites thereof (beam sensitive, $10^{4} \mathrm{e} / \AA^{2}$ ), and for inorganic materials (least beam sensitive, $\left.10^{6} \mathrm{e} / \AA^{2}\right)$. For each projection in the tilt series, an equal incoming electron $I_{0}$ dose is assumed, i.e., $I_{0}=I_{\text {total }} / N$, where $N$ is the number of projections, thus, not taking a dose distribution factor into account. To investigate the influence of the angular sampling scheme, three different tilt increments $\Delta \theta_{0}$ are studied, i.e. $1^{\circ}$ and $5^{\circ}$ constant increments, and Saxton scheme of $3^{\circ}$ as starting increments at zero degree tilt angle [51]. The maximum tilt range is $\pm 65^{\circ}$, which is commonly used in ET. The main acquisition parameters are listed in Table 1 .

The resulting nine tilt-series were reconstructed by SIRT, TVM and DART. In the SIRT reconstructions, the number of iteration was 10,50 , and 100 for accumulated electron dose of $10^{2} \mathrm{e} / \AA^{2}, 10^{4} \mathrm{e} / \AA^{2}$, and $10^{6} \mathrm{e} / \AA^{2}$, respectively. More details on SIRT reconstructions and 
Table 1

TEM parameters and acquisition schemes used for calculating projections.

\begin{tabular}{ll}
\hline Parameter & Value \\
\hline Acceleration voltage $(\mathrm{keV})$ & 300 \\
Underfocus $(\mu \mathrm{m})$ & -5 \\
Total electron dose $\left(\mathrm{e} / \AA^{2}\right)$ & $10^{2}, 10^{4}, 10^{6}$ \\
Tilt range & $\pm 65^{\circ}$ \\
Tilt increment & Constant increment: $1^{\circ}, 5^{\circ}$, Saxton scheme: $3^{\circ}$ \\
\hline
\end{tabular}

how the iteration number was selected can be found in the Supplementary information, Section 1.3. In the TVM approach, the regularization parameter $\mu$ was optimized for each reconstruction based on visual inspection. This is by no means the best approach but commonly done as better defined criteria are often lacking [52]. The standard DART algorithm is utilized with 100 iterations for all the reconstructions [22,40], and a global threshold is used for segmentation which is set to the average value $(0.5)$ of the background ( 0 ) and the particle intensity (1). The volume size for each reconstruction is $508 \times 508 \times 200$ voxels.

\subsubsection{Evaluation methods}

Several methods have been proposed to estimate the resolution in ET but no straightforward widely accepted approach exists [53-57]. If the reconstruction is from a tilt series with a tilt range of $\pm 90^{\circ}$ around a single-tilt axis ( $Y$ direction), the attainable resolution has been estimated to $d_{X}=\pi D / N$ along the $X$ direction [53], where $D$ is the diameter of the object and $N$ is the number of projections. Nevertheless, the limited tilt range in the ET leads to the elongation along the beam direction $Z$ in the reconstruction. Therefore, the resolution in the $Z$ direction $d_{Z}$ is degraded by an elongation factor $e_{x z}$ which is a function of the maximum tilt range $\alpha$ [58]

$d_{z}=d_{x} e_{x z}=d_{x} \sqrt{\frac{\alpha+\sin \alpha \cos \alpha}{\alpha-\sin \alpha \cos \alpha}}$

Obviously, above descriptions of the resolution is only based on sampling considerations. Since noise and blurring also influence the achievable resolution in the reconstruction, they need to be considered as well.

In the present work, edge intensity profiles that encode the effects of dose, imaging and processing have been used to determine the resolution and, thus, the quality of the reconstruction [36,56]. An edge intensity profile is taken across the background-object edge transition with equal numbers of pixels on each side, which is 20 pixel long and 1 pixel wide (Fig. 2). The intensities of the ideal edge profile (Fig. 2b-Ideal) in the model only contain two gray levels: background (0) and objects (1), and the transition occurs at the 10.5 pixel position, referred to as the edge position. These ideal profiles are broadened and become noisy after reconstruction (Fig. 2b-Local). More detailed information about the reconstruction, such as the dynamic range $a$, the background intensity $b$, the edge position $x_{0}$, and the edge steepness $k$, can be obtained from characterization of the reconstructed edge by looking at the reconstructed profiles. To quantify the edge profiles, an edge spread function (ESF) is defined including the above mentioned four parameters. [36,56] Mathematically, it can be represented by a continuous sigmoidal function [59],

$S(x)=\frac{1}{2} a\left[1+\left(\tanh \left(k\left(x-x_{0}\right)\right)\right)\right]+b$

In order to find the four unknown parameters, the ESF is fitted to the edge profiles. The resolution in the reconstruction can be defined by the full-width-at-half-maximum (FWHM) of the first derivative of the edge profiles, which is the inverse of the steepness $k: \Delta r=1.76 / k$. In other words, the steepness $k$ is the a

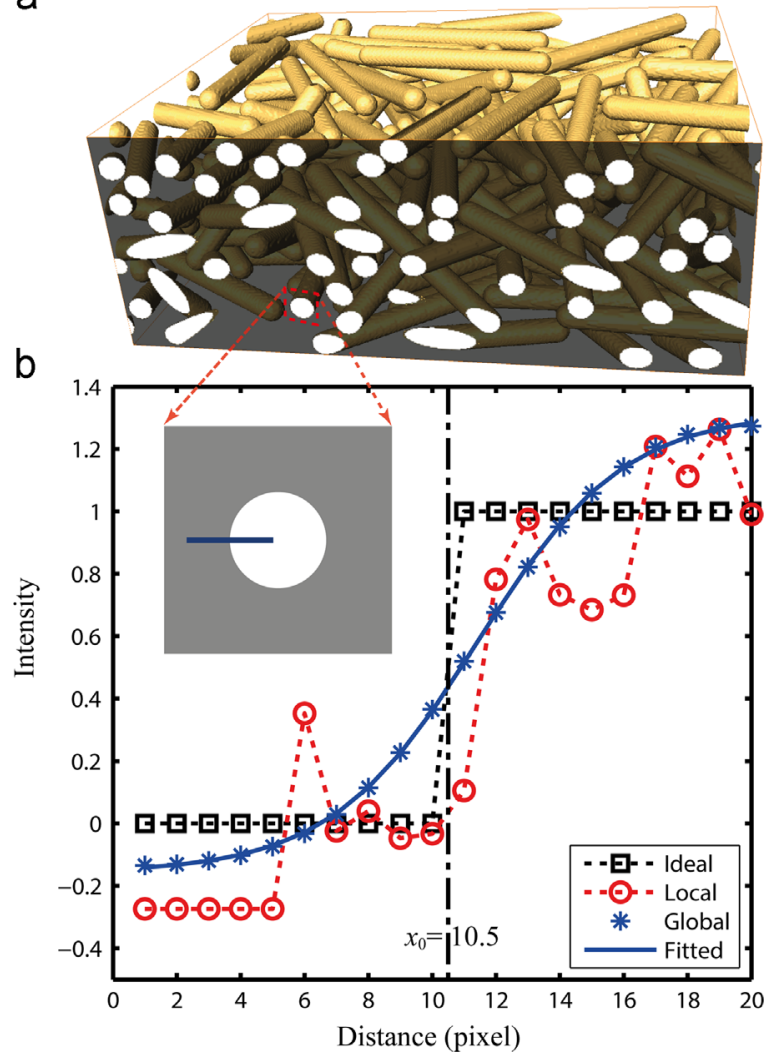

Fig. 2. Illustration of the edge intensity profile from the model, which is taken across the background-object interface. An ideal edge intensity profile is a step profile, 20 pixels long and 1 pixel wide, thus the edge position is at 10.5 pixe (indicated by the middle vertical line). A local edge profile is taken from the reconstruction, and a global edge profile is obtained by averaging all the local edge profiles in the same direction. An edge spread function is fitted to the edge profiles to evaluate the quality of the reconstruction.

resolution in the frequency space. In order to assess the reconstruction quality globally and locally, we define two types of edge profiles: a local edge profile which is an individual edge intensity profile taken directly from a reconstruction with 20 pixel length and 1 pixel width, and a global edge profile which is obtained by averaging all the local edge profiles in the same direction in a reconstruction.

Apart from particle size, the connectivity of objects throughout a volume is of great interest, as it is often closely related to the functional properties of specimens, e.g., conductive network of CNT's in the polymer nanocomposites. Henceforth, we carry out such an analysis and study the resolvable separation between particles by comparing the intensity profiles across gaps of varying size, referred to as gap intensity profiles.

A gap intensity profile is a line intensity profile which is taken from one particle over the background to another particle, as depicted in Fig. 3. Using a similar procedure as for obtaining the global edge profile, a global gap profile is calculated by averaging over all local gap profiles with the same gap width taken from a reconstruction in the same direction. In this manner, each reconstruction yields three global gap profiles, along the $X, Y$ and $Z$ directions, respectively. In order to find the resolvable separation, an inverted Gaussian function is fitted to the global gap profiles, and the gap width is determined by the full-width-at-half-maximum (FWHM) of the fitted Gaussian. To probe for actually resolvable distances between two particles in a reconstruction, four different gap widths were chosen, i.e. $3 \mathrm{~nm}, 6 \mathrm{~nm}, 9 \mathrm{~nm}$, and $12 \mathrm{~nm}$. 


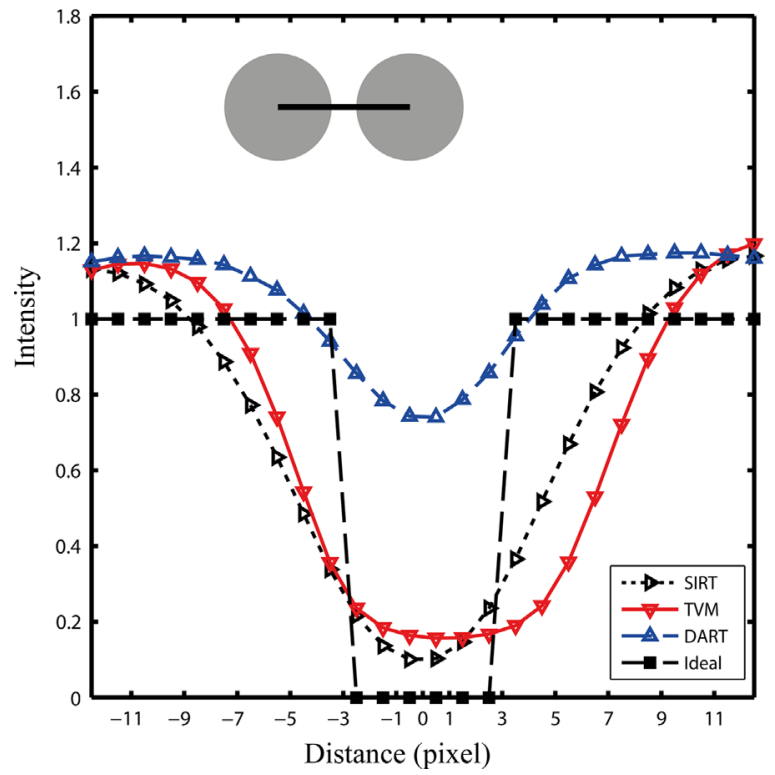

Fig. 3. Illustration of the gap intensity profile, which is taken from one particle side over a background to another particle side. Besides the ideal gap profile with a 6 pixel gap from the model (square), an example global gap profile for a SIRT (right triangle), TVM (down triangle), and DART (up triangle) reconstruction along the $X$ direction are shown.

\section{Results}

In the following, we present visual inspection (3.1), evaluation based on global edge profiles (3.2), local edge profiles (3.3), and gap profiles (3.4). The projections were also reconstructed using WBP as reference for quantitative analysis. Further details on the WBP parameters can be found in the Supplementary information, Section 1.3. For the purpose of visualization, all the reconstructions are normalized to the same mean density. The normalization method is presented in the Supplementary information, Section 2.1.

\subsection{Reconstruction results}

The fidelity of the reconstructions is first visually inspected in comparison with the model. As shown in Fig. 4, there are no significant differences between SIRT, TVM and DART reconstructions at a cumulative electron dose of $10^{2} \mathrm{e} / \AA^{2}$. All reconstructions (Figs. 4 and S3) suffer from high noise contributions, leading to low visibility of the rods. Here SIRT and DART show no directionality of features in the XY plane while TVM shows a characteristic horizontal striping which is a result of breaking up the full 3D dataset into $N \times 2$ D reconstructions. Although the white bands on the left and right of the numerical TVM cross-sections do change the absolute intensity of the features, they do not affect the quantification. (See Supplementary information, Sections 2.1 and 2.2 for a discussion of the effects on quantification).

As expected, reconstructions are notably improved by increasing the total electron dose to $10^{4} \mathrm{e} / \AA^{2}$ (Fig. 5) and $10^{6} \mathrm{e} / \AA^{2}$ (Fig. S4). Compared with the model, it can be seen that particles in the SIRT reconstruction (Fig. 5b) are elongated due to the missing wedge, but in the TVM reconstruction (Fig. 5c) the tubular domains seem thinned, a point that will be further discussed in Section 3.2.2. Moreover, neither SIRT nor TVM algorithms can properly resolve objects whose long axis is perpendicular to the beam direction $Z$ and the tilt-axis $Y$. The same holds true for the DART algorithm where objects oriented along the $X$ axis are sometimes but not always reconstructed (unstable behavior). Nevertheless the DART algorithm significantly suppresses the elongation artifact along the beam direction (Fig. 5d) and separates neighboring objects well. A 3D volume rendering of these results is shown in Fig. S5 in the Supplementary Information, illustrating the advantages of TVM and DART on account of the lowered noise in the reconstruction.

\subsection{Global edge profiles}

In the following, the transitions between tubular domains and matrix in SIRT, TVM, and DART reconstructions will be quantitatively evaluated. Utilizing the edge spread function, we first quantify the quality of reconstructions globally. In each reconstruction, three global edge profiles along the $X, Y$, and $Z$ directions are calculated by averaging all the local edge intensity profiles along the same direction. Subsequently, the edge spread function given by Eq. (3) is fitted to all the global edge profiles. The global edge profiles and the corresponding fitted profiles along both the $X$ and $Z$ directions are plotted and presented in Figs. S6 and S7. The standard errors of the steepness and the edge position and the goodness-of-fit $\left(R^{2}\right)$ are listed in Table S2.

Fig. 6 shows the results of the fitting process focusing on the edge shift $\left(\Delta x_{0}=x_{0}-10.5\right)$ and the steepness $(k)$ as a function of tilt increments and dose. For completeness, the corresponding results using WBP are also presented in Fig. 6. For convenience, the edge position $x_{0}$ is replaced by the edge shift $\Delta x_{0}$, which is the displacement of the edge position in the reconstruction as compared to the model. A negative edge shift means the edge moves to the background side which leads to an increase in object volume, while a positive edge shift indicates that the edge moves into the object side which results in a decrease of the object volume. In the following, we will discuss the results for each algorithm in terms of dose and the acquisition scheme.

\subsubsection{SIRT}

As shown in Fig. $6 a$ and $b$, the edge position in the SIRT reconstructions along the $X$ direction are positively shifted $(0.5-$ 1.0 ), whereas along the $Z$ direction are negatively shifted $(-0.5$ to -1.0 ) for all the acquisition schemes. Despite of the measured edge shifts, effects of the total electron dose and tilt increments are absent, rendering the algorithm insensitive and stable with respect to changing acquisition conditions. The steepness $k$ in SIRT reconstruction generally increases from $0.17\left(10^{2} \mathrm{e} / \AA^{2}\right)$ to 0.21 $\left(10^{4} \mathrm{e} / \AA^{2}\right)$ to $0.23\left(10^{6} \mathrm{e} / \AA^{2}\right)$ along the $X$ direction, while from 0.13 $\left(10^{2} \mathrm{e} / \AA^{2}\right)$ to $0.16\left(10^{4} \mathrm{e} / \AA^{2}\right)$ to $0.17\left(10^{6} \mathrm{e} / \AA^{2}\right)$ along the $Z$ direction, as shown in Fig. $6 \mathrm{c}$ and $\mathrm{d}$. This dose effects become clearer when looking at the spatial resolution $\Delta r$ which changes, for example, from $13.5 \mathrm{~nm}\left(10^{2} \mathrm{e} / \AA^{2}\right)$ to $11.1 \mathrm{~nm}\left(10^{4} \mathrm{e} / \AA^{2}\right)$ to $10.4 \mathrm{~nm}\left(10^{6} \mathrm{e} / \AA^{2}\right)$ along the $Z$ direction. At equal total electron doses, the steepness $k$ in the $X$ direction is independent of the tilt increments (Fig. 6c). Along the $Z$ direction (Fig. 6d) slight variations are observed such that the resolution using a Saxton $3^{\circ}$ tilt-scheme is approximately $0.5 \mathrm{~nm}$ better than using $1^{\circ}$ increment, and $1 \mathrm{~nm}$ better than using $5^{\circ}$ increments. The elongation factor $e_{x z}$ determined from the $z$ vs. $x$ size of the rods at the electron dose $10^{2} \mathrm{e} / \AA^{2}$ is about 1.25 , and 1.37 at both the electron dose of $10^{4} \mathrm{e} / \AA^{2}$ and $10^{6} \mathrm{e} / \AA^{2}$, less than the value predicted for the elongation of the WBP point-spread function i.e. $e_{x z}=1.42$ [58].

\subsubsection{TVM}

In the TVM reconstructions, the measured edge positions are strongly influenced by the tilt increments and the total electron dose (Fig. 6 a and b). Similar as for SIRT, at a total electron dose of $10^{2} \mathrm{e} / \AA^{2}$, the edge position in the $X$ direction is positively shifted, while in the $Z$ direction it is negatively shifted. In both directions, the edge shifts are less than $1.0 \mathrm{~nm}$, regardless of the tilt 

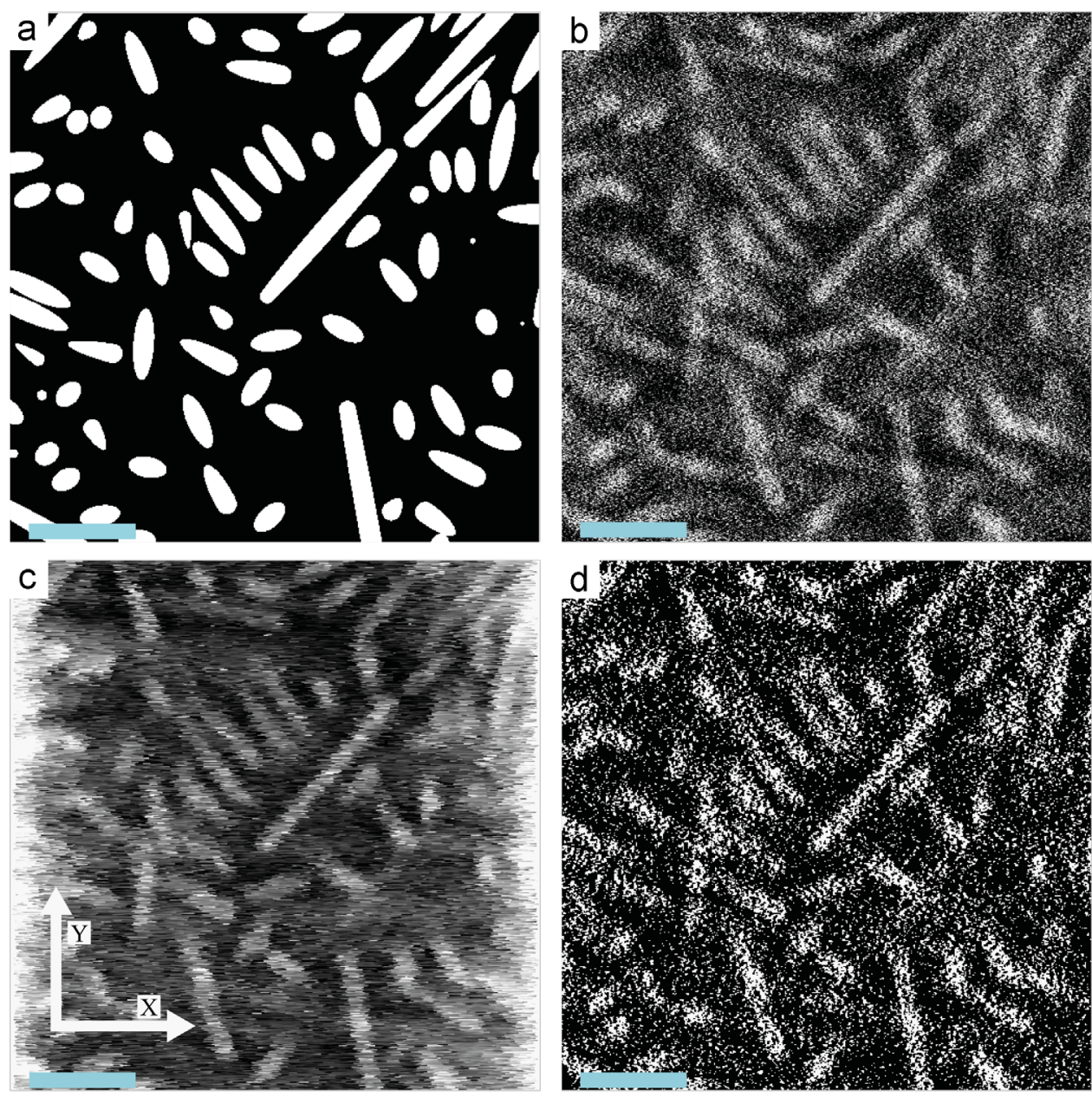

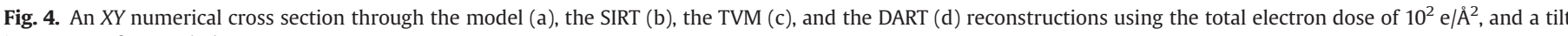
increment of $1^{\circ}$. Scale bars are $100 \mathrm{~nm}$.

increments. For higher electron doses, the edge position is still positively shifted but further away from the original position in the $X$ direction, whereas in the $Z$ direction the edge shift becomes positive. It is interesting that the edge shift is increasing with increasing tilt increments in both $X$ and $Z$ directions, indicating that the compressed sensing approaches is significantly influenced by the tilt increment when a limited electron dose is used. This is contrary to previous results where compressive sensing based approaches are used for the reconstruction of nanostructures based on high-angle annular dark-field STEM tilt series with a higher SNR [23,35]. As a consequence of the observed edge shifts, the size of objects will be underestimated. For example, the volume of a sphere with a $10 \mathrm{~nm}$ radius will be reduced by $20 \%$, taking the edge shift from the reconstruction at the total electron of $10^{4} \mathrm{e} / \AA^{2}$ with the Saxton $3^{\circ}$ tilt increments into account. However, it must be noted that this underestimation may be largely influenced by a non-optimal choice of the regularization parameter. Changing this parameter will yield large differences and may even result in an overestimation of the observed edge shift. Therefore, obtaining an objective approach for determining the optimal penalty parameter may be crucial in order to obtain accurate quantitative results.

The steepness, i.e. resolution, in the TVM reconstruction mainly depends on the total electron dose (Fig. $6 \mathrm{c}$ and d), improved from 0.18 to 0.30 in the $X$ direction and from 0.15 to 0.28 in the $Z$ direction for $10^{2} \mathrm{e} / \AA^{2}$ and $10^{4} \mathrm{e} / \AA^{2}$, respectively. Only minor improvements in steepness are observed from $10^{4} \mathrm{e} / \AA^{2}$ to $10^{6} \mathrm{e} / \AA^{2}$. TVM, as for SIRT and WBP, shows only slight changes with tilt increments, and the values for $X$ and $Z$ only slightly differing resulting in a rather isotropic resolution for this limited $\pm 65^{\circ}$ tilt-range.

\subsubsection{DART}

The observed edge positions in DART reconstructions are mainly related to the total electron dose (Fig. 6a and b). At the electron dose of $10^{2} \mathrm{e} / \AA^{2}$, the edge position in the $X$ direction moves to the inside of the object and the shift slightly increases from $1.03 \mathrm{~nm}$ to $1.15 \mathrm{~nm}$ to $1.36 \mathrm{~nm}$ as the tilt increments change from $1^{\circ}$, to Saxton $3^{\circ}$, to $5^{\circ}$. It is interesting that, however, the edge shift in the $Z$ direction is negligible, $-0.17-0.1$, which indicates the elongation effect owing to the missing wedge has been suppressed. At total electron doses of $10^{4} \mathrm{e} / \AA^{2}$ and $10^{6} \mathrm{e} / \AA^{2}$, positive edge shifts in the both $X$ and $Z$ directions are observed. The edge position in the $X$ direction is close to the original position, whereas in the $Z$ direction it moves $\sim 0.85 \mathrm{~nm}$ to the inside of the object, as shown in Fig. 6a and b. Overall, only a minor influence of the tilt increments on the edge positions are found.

While individual edge profiles in the DART reconstruction are step profiles on account of the binarized reconstruction, the global edge profile nevertheless is an average of overall local edge profiles, and thus the global steepness mainly represents uncertainty in local edge positions. Hence, as displayed in Fig. $6 \mathrm{c}$ and d, a steepness of the global edge smaller than 0.8 is obtained. This is 

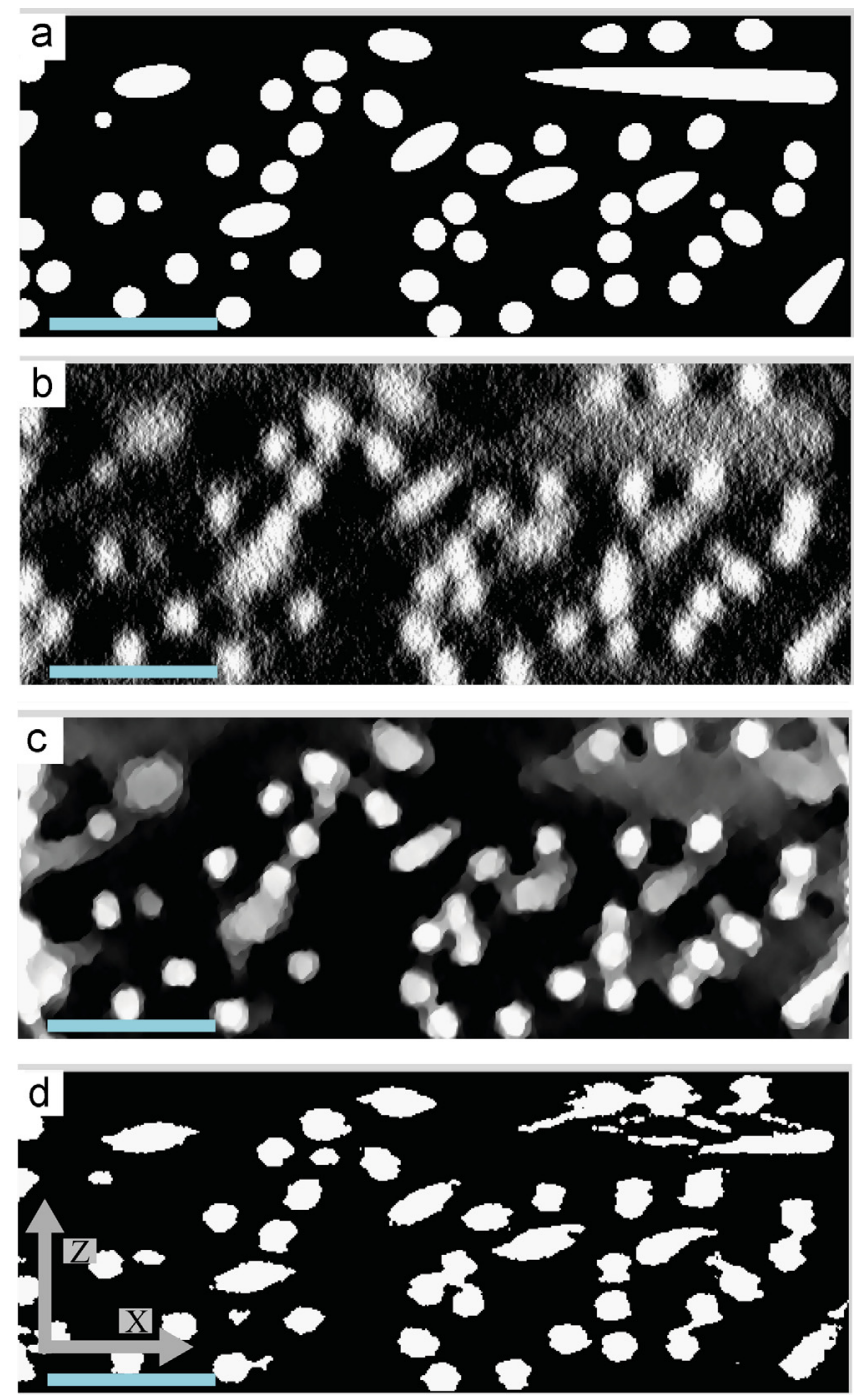

Fig. 5. An $X Z$ numerical cross section through the model (a), the SIRT (b), the TVM (c), and the DART (d) reconstructions using the total electron dose of $10^{4} \mathrm{e} / \AA^{2}$ and a tilt increment of $1^{\circ}$. Scale bar is $100 \mathrm{~nm}$.

significantly larger than for all other reconstruction approaches and highlights the uniqueness of the method. We will discuss the distribution of local edge positions in the next section.

\subsection{4. $W B P$}

From Fig. 6, it can be seen that both the edge position and the steepness of the global edge profiles from the WBP reconstruction are barely influenced by the changes of the total electron dose and the sampling schemes. The reason is that the procedure of gaining the global edge profiles by averaging over approximately a million edge profiles leads to virtually noise-free global edge profiles, though the SNR of the reconstruction strongly varies with changes of the total electron dose. The edge shifts in the $X$ direction are negligible, whereas in the $Z$ direction they are shifted to the background side. As expected, the steepness along the $X$ direction (ca. 0.21) is larger than along the $Z$ direction (ca. 0.17). The resulting elongation factor, i.e. $e_{x z}=1.21$, is smaller than the theoretical value for the WBP PSF (1.42). Overall, the performance of WBP is in line with more advanced approaches looking at the global edge profile alone. For more details about the performance of the WBP reconstruction in terms of the limited electron dose and the acquisition schemes, we refer to Ref. [36].

\subsection{Local edge profile}

Since the global edge profiles are averages of all local edge profiles, characterization of such profiles mainly reveals overall trends in reconstruction quality. Nevertheless, dose related properties are mainly encoded in the local edge profiles. To gain further knowledge about the behavior of the three reconstruction algorithms in terms of the total electron dose, we focus in this section on the statistical characterization of local edge profiles. To this end, first an edge spread function is fitted to the local edge profiles along the $X, Y$, and $Z$ directions separately. Second, the probability density distributions of the edge shifts from each reconstruction are obtained and analyzed statistically. Note that it is not appropriate to compare the steepness in the DART reconstruction with the steepness in the SIRT and the TVM reconstruction, as the individual edge profile in the DART reconstruction is a binary profile and therefore the steepness is infinitely large. A detailed description of the fitting procedure can be found in the Supplementary information, Section 2.4 and Fig. S8.

Fig. 7 shows the probability density distribution of edge shifts along the $X$ direction in the SIRT, TVM, DART, and WBP reconstructions, whereas Figs. S9 and S10 present the corresponding distributions along the $Z$ and $Y$ direction, respectively. The corresponding population mean, standard error of population mean and standard deviation are shown in Table S3. Since the standard error of the population mean is significantly smaller than the differences in edge shift between different reconstructions (see Table S3), observed differences in edge shift are significant and interpretable. In general, it is observed that the probability distribution of the edge shifts in each reconstruction becomes wider (increasing standard deviation) with a decreasing total electron dose.

In the SIRT reconstruction, at an electron dose of $10^{6} \mathrm{e} / \AA^{2}$ (Fig. 7a), the distribution of edge shifts in each direction is a quasiGaussian distribution which its mean closes to the corresponding global edge shift. Compared to the distribution of the edge shift in the $X$ direction with the standard deviation $\sigma_{x} \approx 1.69 \mathrm{~nm}$, the distribution in the $Z$ direction (Fig. S9a) is considerably wider $\left(\sigma_{z} \approx 2.30 \mathrm{~nm}\right)$, reflecting the effect of the missing wedge. As the total electron dose decreases to $10^{4} \mathrm{e} / \AA^{2}$ (Fig. 7b), the probability distribution of the edge shifts is still a quasi-Gaussian distribution but broadened further, e.g., with $\sigma_{x} \approx 2.23 \mathrm{~nm}$ standard deviation for the $X$ direction. At the lowest electron dose of $10^{2} \mathrm{e} / \AA^{2}$ (Fig. 7c), however, the probability distribution of the edge shifts becomes a uniform distribution, leading to a large variation in determination of edge positions, e.g. $\sigma_{x}=3.50 \mathrm{~nm}$. Consequently, the reliability of measurements, such as object size, will be very low. For instance, assuming a sphere of $20 \mathrm{~nm}$ in diameter reconstructed at a total dose of $10^{2} \mathrm{e} / \AA^{2}$, and considering the uncertainty along both $X, Y$ and $Z$ direction given by the standard deviation of the distribution, will result in sphere volumes between $20 \%$ and $220 \%$ of the initial value! Again, only minor influence of the tilt increments on the edge positions and the steepness has been observed, which is consistent with the results from global edge profiles.

In the TVM reconstruction, the probability distribution of edge positions at the total electron dose of $10^{6} \mathrm{e} / \AA^{2}\left(\sigma_{x} \sim 2.06\right)$ is similar to the distribution at the electron dose of $10^{4} \mathrm{e} / \AA^{2}\left(\sigma_{x} \approx 2.08 \mathrm{~nm}\right)$, as shown in Fig. $7 d$ and e. However, it becomes significantly widened $\left(\sigma_{x} \approx 3.35 \mathrm{~nm}\right)$ as the electron dose reduces to $10^{2} \mathrm{e} / \AA^{2}$ (Fig. 7f). In addition, the variation of edge shifts in the reconstructions at the total electron dose of $10^{4} \mathrm{e} / \AA^{2}$ and $10^{6} \mathrm{e} / \AA^{2}$ slightly increases as the tilt increments become larger. For instance, the mean and standard deviation in Fig. 7e change from $0.75 \pm 1.95 \mathrm{~nm}$, 
a

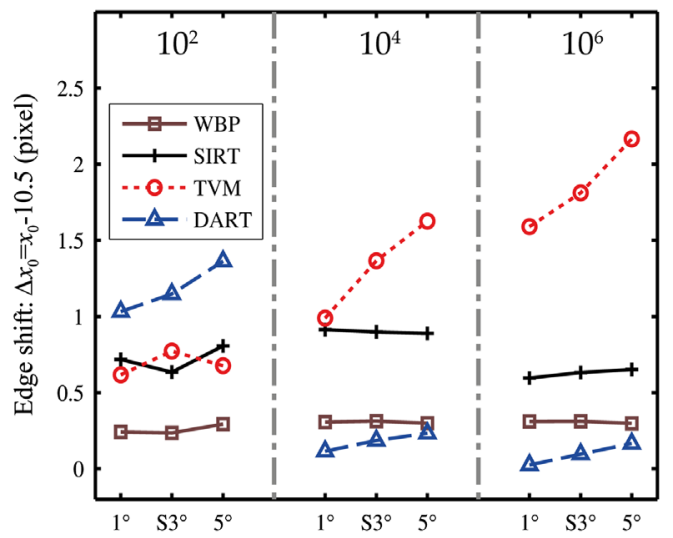

C

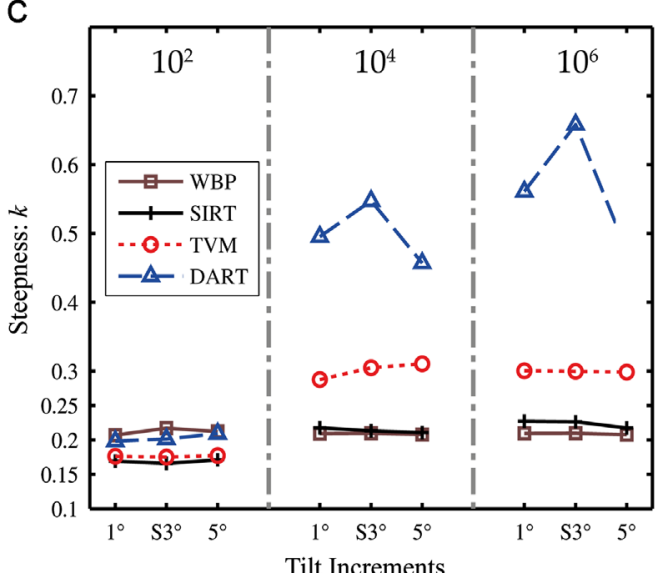

b

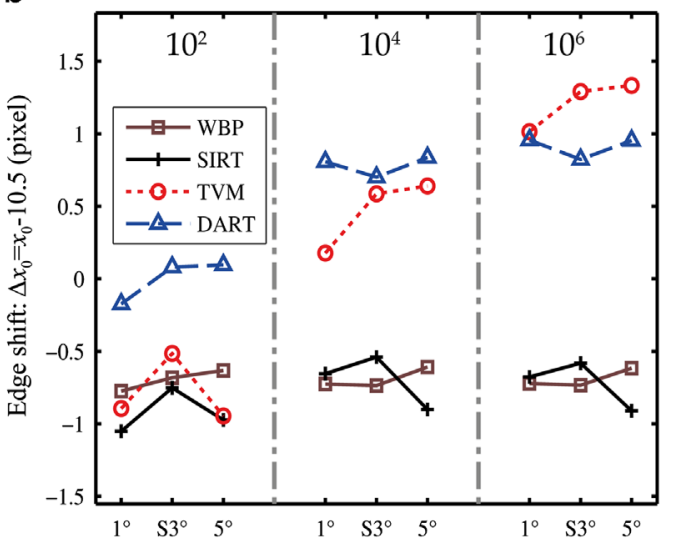

d

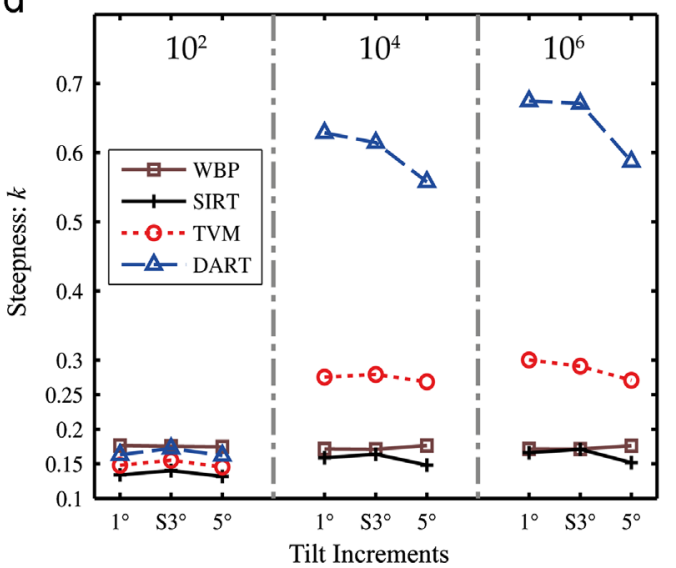

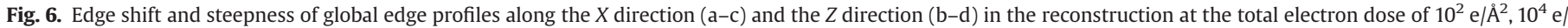

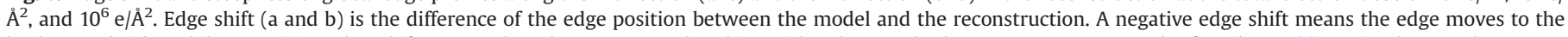

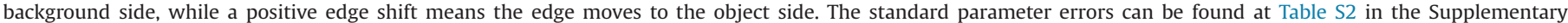
Information. S3 ${ }^{\circ}$ denotes the Saxton scheme with an angular starting interval of $3^{\circ}$.

via $1.05 \pm 2.06 \mathrm{~nm}$, to $1.23 \pm 2.22 \mathrm{~nm}$ as increase of the tilt increments from $1^{\circ}$, via Saxton $3^{\circ}$, to $5^{\circ}$.

In the DART reconstruction, the probability distribution of edge shifts is slightly widened as the total electron dose decreases from $10^{6} \mathrm{e} / \AA^{2}$ to $10^{4} \mathrm{e} / \AA^{2}$, though the mean edge shifts are similar (Fig. $7 \mathrm{~g}$ and h). Nevertheless, at the total electron dose of $10^{2} \mathrm{e} / \AA^{2}$, it is not only broadened but also significantly shifted into the object side, e.g. $1.40 \pm 2.83 \mathrm{~nm}$ along the $X$ direction (Fig. $7 \mathrm{i}$ ). In addition, the tilt increments have an influence on the distribution of edge positions at a total electron dose of $10^{4} \mathrm{e} / \AA^{2}$ and $10^{6} \mathrm{e} / \AA^{2}$. Here, the variation using the Saxton $3^{\circ}$ angular scheme has the smallest and $5^{\circ}$ constant increments the largest spread, i.e. $\sigma_{x}=1.67 \mathrm{~nm}$ and $\sigma_{x}=2.02 \mathrm{~nm}$ in Fig. $7 \mathrm{~g}$, respectively. It should be pointed out that the influence of tilt increments is negligible in the reconstruction at a total electron dose of $10^{2} \mathrm{e} / \AA^{2}$, as can be seen in Fig. $7 \mathrm{i}$.

For the WBP reconstruction, Fig. 7 shows that the distribution of edge positions is dramatically broadened as the decrease of the total electron dose. For example, the standard deviation of the edge shifts in $Z$ direction changes from $2.44 \mathrm{~nm}$, via $3.68 \mathrm{~nm}$, to $4.14 \mathrm{~nm}$ as the total electron dose reduces from $10^{6} \mathrm{e} / \AA^{2}$, via $10^{4} \mathrm{e} / \AA^{2}$, to $10^{2} \mathrm{e} / \AA^{2}$. It should be noted that only minor influence of the tilt increments on the distribution of edge positions has been observed, as displayed in Fig. $7 \mathrm{j}-1$.

\subsection{Gap profile}

Fig. 8 presents the difference $\Delta w$ between the measured gap width $w_{\mathrm{m}}$ and the actual gap width $w_{\mathrm{a}}$ along both the $X$ and $Z$ direction, i.e., $\Delta w=w_{\mathrm{m}}-w_{\mathrm{a}}$. A negative value $(\Delta w<0)$ represents a narrowed gap whereas positive values $(\Delta w>0)$ represent a broadened gap. Here we note that some global gap profiles in the $Z$ direction contain such low intensity variations that the Gaussian could not be fitted, thus leading to blank data fields in Fig. 8. Overall, there is a similar trend for all algorithms, namely an increased precision with increasing gap width. This effect is best seen at a total electron dose of $10^{6} \mathrm{e} / \AA^{2}$ (Fig. 8a and b) where the measured gap width in the SIRT, TVM, and WBP reconstructions converge towards the model when the gap increases from $3 \mathrm{~nm}$ to $12 \mathrm{~nm}$. Generally, $3 \mathrm{~nm}$ and $6 \mathrm{~nm}$ gaps are overestimated using the three reconstruction methods. In the SIRT reconstruction, the smallest difference between the ideal and measured gap is found in the $12 \mathrm{~nm}$ gap, i.e., $\Delta w_{x}=0.5 \mathrm{~nm}$ and, $\Delta w_{z}=-0.5 \mathrm{~nm}$. The $9 \mathrm{~nm}$ gaps in the WBP are well determined, whereas the $12 \mathrm{~nm}$ gaps are slightly underestimated. In the TVM reconstruction, the most accurate result has been found for the $12 \mathrm{~nm}$ gaps, but one needs to consider that the measured value strongly depends on the applied tilt scheme (especially along the $X$ direction Fig. 8a). In contrast, DART is the most accurate at small gap widths and more significantly underestimates $9 \mathrm{~nm}$ and $12 \mathrm{~nm}$ gaps. As can be seen in Fig. 8c and d, similar measurements of gap widths have been obtained for the reconstruction at a total electron dose of $10^{4} \mathrm{e} / \AA^{2}$. Here we like to point out that the $3 \mathrm{~nm}$ gaps in the $Z$ direction are barely resolved in the SIRT and WBP reconstructions (Fig. 8b and d).

In all the reconstructions at electron doses of $10^{2} \mathrm{e} / \AA^{2}$ (Fig. 8e), the measured gap value converges to the model value when going from $3 \mathrm{~nm}$ to $12 \mathrm{~nm}$ along the $X$ direction. It is found that the most 

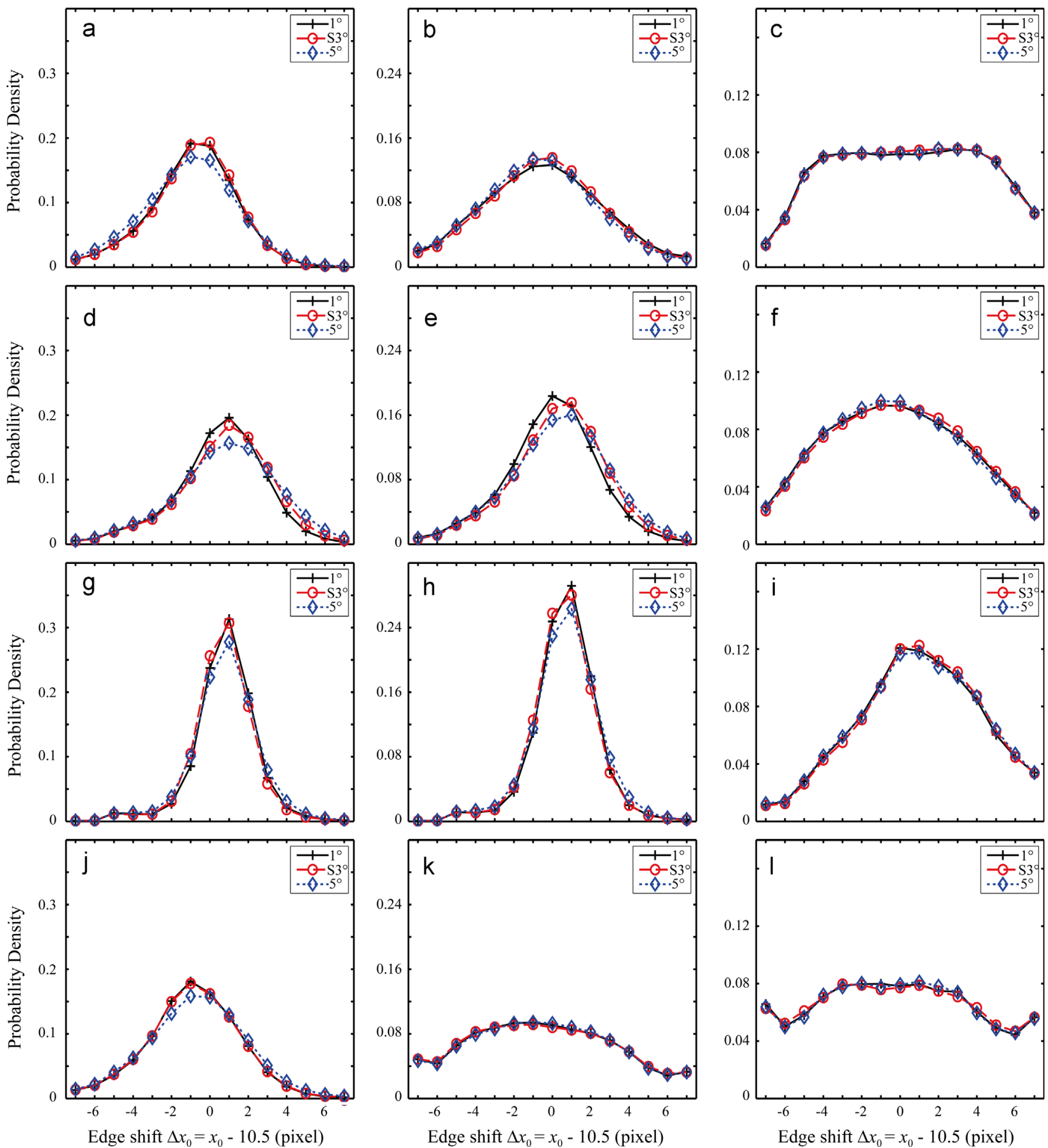

Edge shift $\Delta x_{0}=x_{0}-10.5$ (pixel)

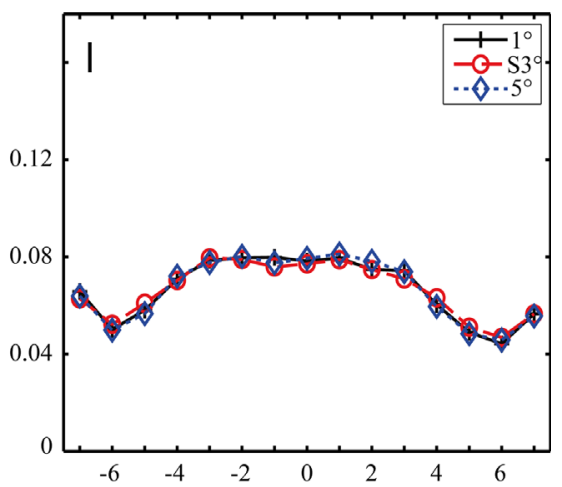

Edge shift $\Delta x_{0}=x_{0}-10.5$ (pixel)

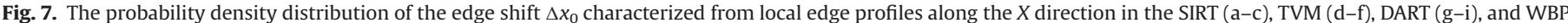
(j-l) reconstruction at a total electron dose $10^{6} \mathrm{e} / \AA^{2}(\mathrm{a}, \mathrm{d}, \mathrm{g}, \mathrm{j}), 10^{4} \mathrm{e} / \AA^{2}(\mathrm{~b}, \mathrm{e}, \mathrm{h}, \mathrm{k})$, and $10^{2} \mathrm{e} / \AA^{2}(\mathrm{c}, \mathrm{f}, \mathrm{i}, \mathrm{l})$, with the tilt increments of $1^{\circ}, 5^{\circ}$, and Saxton $3^{\circ}$.

accurate determined measurement is for the $9 \mathrm{~nm}$ gaps in the WBP. Nevertheless, $3 \mathrm{~nm}$ gaps in the WBP reconstruction along the $X$ direction cannot be resolved because of too noisy data. Along the $Z$ direction, only $9 \mathrm{~nm}$ and $12 \mathrm{~nm}$ gaps can be determined from all the reconstructions, while the $6 \mathrm{~nm}$ gaps can only be found in both TVM and DART reconstruction, or with SIRT and WBP but using a Saxton $3^{\circ}$ tilt scheme. Three nanometer gaps along the $Z$ direction cannot be determined from any reconstruction at the total electron dose of $10^{2} \mathrm{e} / \AA^{2}$.

\section{Discussion and conclusion}

Although the SIRT, TVM and DART reconstruction methods have been compared before in the literature [22,23,32,33], these studies are generally based on experimental data, lacking a detailed knowledge of the ground truth. In this work, we have simulated the electron tomography workflow based on a welldefined model of tubular domains in a matrix with slab geometry. The model was chosen as it closely resembles a large range of 
a

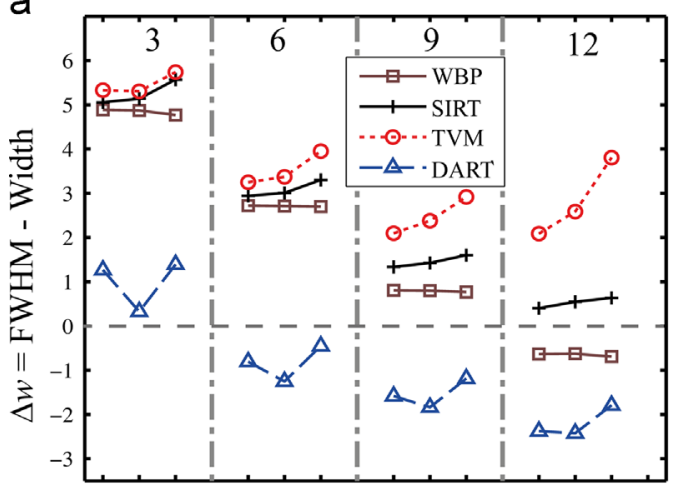

C

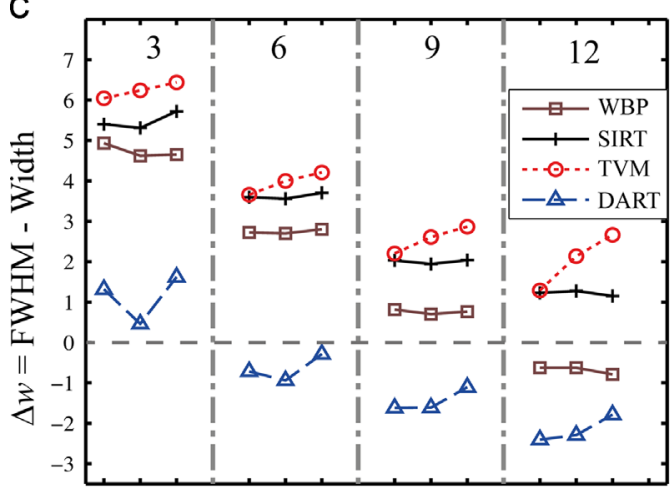

e

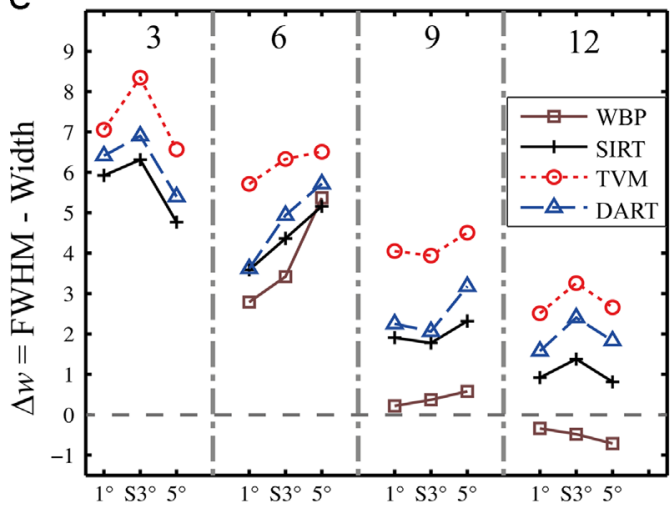

Tilt Increments b

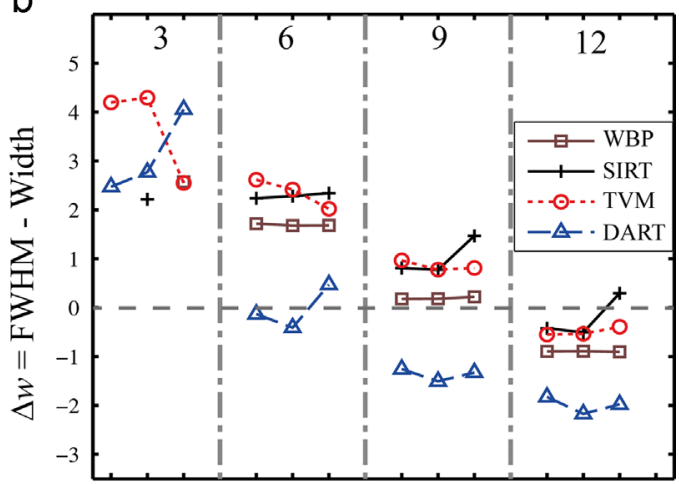

d

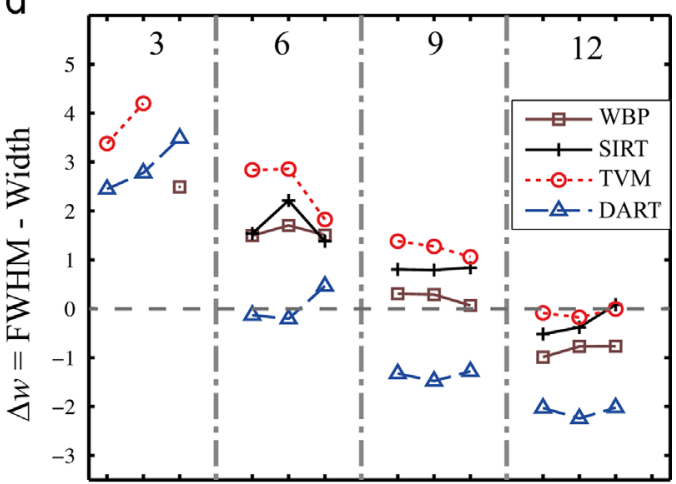

$f$

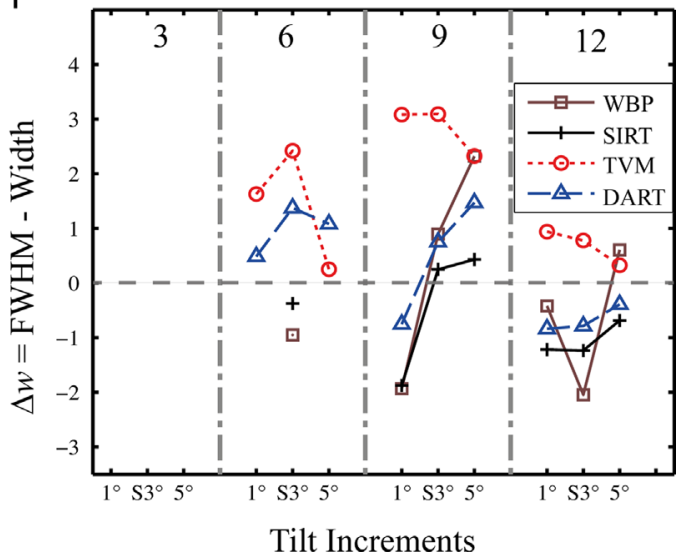

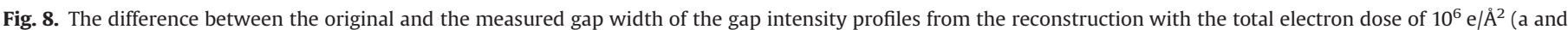

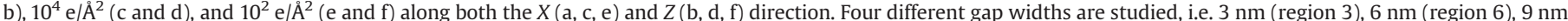

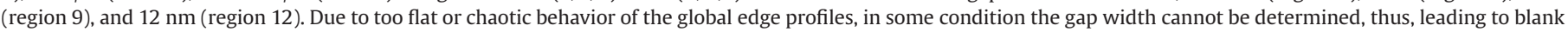
data points, for example, for the $3 \mathrm{~nm}$ gaps along the $Z$ direction (f). $53^{\circ}$ denotes the Saxton scheme with an angular starting interval of $3^{\circ}$.

functional materials, such as a P3HT/PCBM bulk heterojunction thin-film or a CNT/polymer nanocomposite thin-section. In particular we investigated effects of a limited total electron dose and variations in tilt scheme on the properties of this three reconstruction methods. Both the advantages and disadvantages of the SIRT, TVM, and DART are quantitatively analyzed by comparing with the ground truth of the model. Our comparisons not only include the sharpness of the transitions between phases which provides an estimate of the achievable resolution, but also the position of interfaces and the size of gaps between objects which are key parameters in quantification of object morphologies and connectivity.

Expectedly, as the electron dose available for imaging decreases, the fidelity of all reconstruction methods decreases. We find that the SIRT algorithm is the least affected by variations in total dose and tilt scheme, which means that the fidelity of the
SIRT reconstruction is preserved when using fewer projections. This is a clear benefit for beam-sensitive specimens and if a large variety of imaging conditions or tilt schemes are utilized. The disadvantage is that the SIRT reconstruction suffers from a low SNR, and thus it needs extra efforts to denoise and segment the tomograms before it can be used for quantification. In contrast, TVM significantly reduces noise and sharpens transitions between materials which enables a simpler and easier segmentation of the reconstruction. However, objects in the TVM reconstruction become thinned as compared to the model, as illustrated by the measured edge shifts and the overestimation of gap sizes (Fig. S11). Moreover, a significant dependence of TVM on the applied tilt scheme is observed, which indicates that with the presented measures a lower number of images (i.e., larger tilt increments) is not advised. This is an especially important point as this study 
cannot reproduce the general conclusions that compressive sensing approaches deal better with fewer projections $[32,33,35]$. The DART provides a binarized reconstruction and suppresses the elongation artifacts along the beam direction.

Here it is noteworthy that, at the total electron dose of $10^{2} \mathrm{e} / \AA^{2}$ the WBP yields a slightly better global resolution in comparison with the SIRT, TVM, and DART method, though it is also the noisiest reconstruction. The poor results for TVM and DART at this total dose are possibly related to the inappropriate $l^{2}$-norm in the data fit term which assumes a Gaussian distribution instead of a Poisson distribution for noise. Finally, one advantage of WBP and SIRT are their easier implementation and use, whereas TVM and DART are sensitive to the reconstruction parameters and require expert knowledge for properly setting regularization parameters or ratio of boundary pixels, respectively. In summary, to determine which of the many possible reconstruction approaches, such as WBP [13], SIRT [26], TVM [23], DART [22], ART [27], EST [60], Directt [61], W-SIRT [62], is best for a particular class of samples, a study as the one presented here for tubular domains in a slab matrix should be carried out. ${ }^{1}$

\section{Acknowledgments}

The authors thank prof. M. Dijkstra and Dr. R. Ni (Utrecht University, the Netherlands) for providing the random packing code, and Maarten Wirix for characterization of CCD noise. This research forms part of the research program of the Dutch Polymer Institute (DPI), projects \#615. This work is supported by the Research Foundation Flanders (FWO Vlaanderen) through a Ph. D. research grant to B.G.

\section{Appendix A. Supporting information}

Supplementary data associated with this article can be found in the online version at http://dx.doi.org/10.1016/j.ultramic.2014.08.005.

\section{References}

[1] H. Jinnai, R.J. Spontal, Transmission electron microtomography in polymer research, Polymer 50 (2009) 1067-1087.

[2] H. Friedrich, P.E. de Jongh, A.J. Verkleij, K.P. de Jong, Electron tomography for heterogeneous catalysts and related nanostructured materials, Chem. Rev. 109 (2009) 1613-1629.

[3] R. Leary, P.A. Midgley, J.M. Thomas, Recent advances in the application of electron tomography to materials chemistry, Acc. Chem. Res. (2012).

[4] P.A. Midgley, R.E. Dunin-Borkowski, Electron tomography and holography in materials science, Nat. Mater. 8 (2009) 271-280.

[5] S.D. Oosterhout, M.M. Wienk, S.S. van Bavel, R. Thiedmann, L.J.A. Koster J. Gilot, J. Loos, V. Schmidt, R.A.J. Janssen, The effect of three-dimensional morphology on the efficiency of hybrid polymer solar cells, Nat. Mater. 8 (2009) 818-824.

[6] S.S. van Bavel, E. Sourty, G. de With, J. Loos, Three-dimensional nanoscale organization of bulk heterojunction polymer solar cells, Nano Lett. 9 (2009) 507-513.

[7] H. Jinnai, R.J. Spontak, T. Nishi, Transmission electron microtomography and polymer nanostructures, Macromolecules 43 (2010) 1675-1688.

[8] S. Bals, S. Van Aert, G. Van Tendeloo, High resolution electron tomography, Curr. Opin. Solid State Mater. Sci. 17 (2013) 107-114.

[9] J.J. Fernandez, Computational methods for materials characterization by electron tomography, Curr. Opin. Solid State Mater. Sci. 17 (2013) 93-106.

[10] L.F. Drummy, C. Kubel, Polymer microscopy: Current challenges, Polym. Rev. 50 (2010) 231-234

\footnotetext{
${ }^{1}$ Additional to the aspects discussed, experimental misalignment including magnification changes, image rotation, residual lateral and axial shifts, microscope and sample holder as well as alignment procedure used, affect the final result in practice. This can be taken into account, e.g. by taking into account an additional (Gaussian) distribution for the shifts and rotations, but since the settings for this are highly specific, we consider these effects as beyond the scope of the present investigation.
}

[11] G. Van Tendeloo, S. Bals, S. Van Aert, J. Verbeeck, D. Van Dyck, Advanced electron microscopy for advanced materials, Adv. Mater. 24 (2012) 5655-5675.

[12] P.A. Midgley, M. Weyland, 3d electron microscopy in the physical sciences: The development of z-contrast and eftem tomography, Ultramicroscopy 96 (2003) 413-431.

[13] M. Radermacher, Weighted back-projection methods, Electron Tomogr. (2006) 245-273.

[14] C. Kubel, D. Niemeyer, R. Cieslinski, S. Rozeveld, Electron tomography of nanostructured materials - towards a quantitative $3 \mathrm{~d}$ analysis with nanometer resolution, Mater. Sci. Forum 638-642 (2010) 2517-2522 (Thermec 2009, Pts1-4), http://dx.doi.org/10.4028/www.scientific.net/MSF.638-642.2517.

[15] H. Friedrich, P.M. Frederik, G. de With, N.A.J.M. Sommerdijk, Imaging of selfassembled structures: Interpretation of tem and cryo-tem images, Angew. Chem. Int. Ed. 49 (2010) 7850-7858.

[16] R.F. Egerton, Control of radiation damage in the tem, Ultramicroscopy 127 (2013) 100-108.

[17] P.K. Luther, Sample shrinkage and radiation damage of plastic sections, Electron Tomogr. (2006) 17-48.

[18] P. Penczek, M. Marko, K. Buttle, J. Frank, Double-tilt electron tomography, Ultramicroscopy 60 (1995) 393-410.

[19] D.N. Mastronarde, Dual-axis tomography: an approach with alignment methods that preserve resolution, J. Struct. Biol. 120 (1997) 343-352.

[20] N. Kawase, M. Kato, H. Nishioka, H. Jinnai, Transmission electron microtomography without the missing wedge for quantitative structural analysis, Ultramicroscopy 107 (2007) 8-15.

[21] X.X. Ke, S. Bals, D. Cott, T. Hantschel, H. Bender, G. Van Tendeloo, Threedimensional analysis of carbon nanotube networks in interconnects by electron tomography without missing wedge artifacts, Microsc. Microanal. 16 (2010) 210-217.

[22] K.J. Batenburg, S. Bals, J. Sijbers, C. Kubel, P.A. Midgley, J.C. Hernandez, U. Kaiser, E.R. Encina, E.A. Coronado, G. Van Tendeloo, 3d imaging of nanomaterials by discrete tomography, Ultramicroscopy 109 (2009) 730-740.

[23] B. Goris, W. Van den Broek, K.J. Batenburg, H.H. Mezerii, S. Bals, Electron tomography based on a total variation minimization reconstruction technique, Ultramicroscopy 113 (2012) 120-130.

[24] W.O. Saxton, W. Baumeister, M. Hahn, Three-dimensional reconstruction of imperfect two-dimensional crystals, Ultramicroscopy 13 (1984) 57-70.

[25] A.C. Kak, M. Slaney, Principles of computerized tomographic imaging, Society for Industrial and Applied Mathematics, Philadelphia, 2001.

[26] P. Gilbert, Iterative methods for 3-dimensional reconstruction of an object from projections, J. Theor. Biol. 36 (1972) 105-117.

[27] R. Gordon, R. Bender, G.T. Herman, Algebraic reconstruction techniques (art) for 3-dimensional electron microscopy and x-ray photography, J. Theor. Biol. 29 (1970) 471-481.

[28] J.J. Fernandez, A.F. Lawrence, J. Roca, I. Garcia, M.H. Ellisman, J.M. Carazo, Highperformance electron tomography of complex biological specimens, J. Struct. Biol. 138 (2002) 6-20.

[29] T.M. Benson, J. Gregor, Modified simultaneous iterative reconstruction technique, for faster parallel computation, IEEE Nucl. Sci. Symp. Conf. Rec. 1-5 (2005) (2005) 2715-2718.

[30] S. Bals, K.J. Batenburg, J. Verbeeck, J. Sijbers, G. Van Tendeloo, Quantitative three-dimensional reconstruction of catalyst particles for bamboo-like carbon nanotubes, Nano Lett. 7 (2007) 3669-3674.

[31] S. Bals, K.J. Batenburg. D.D, Liang, O. Lebedev, G. Van Tendeloo, A Aerts, J.A. Martens, C.E.A. Kirschhock, Quantitative three-dimensional modeling of zeotile through discrete electron tomography, J. Am. Chem. Soc. 131 (2009) $4769-4773$.

[32] Z. Saghi, D.J. Holland, R. Leary, A. Falqui, G. Bertoni, A.J. Sederman, L.F. Gladden, P.A. Midgley, Three-dimensional morphology of iron oxide nanoparticles with reactive concave surfaces. A compressed sensing-electron tomography (cs-et) approach, Nano Lett. 11 (2011) 4666-4673.

[33] B. Goris, T. Roelandts, K.J. Batenburg, H.H. Mezerji, S. Bals, Advanced reconstruction algorithms for electron tomography: from comparison to combination, Ultramicroscopy 127 (2013) 40-47.

[34] A.H. Andersen, A.C. Kak, Simultaneous algebraic reconstruction technique (sart) a superior implementation of the art algorithm, Ultrason. Imag. 6 (1984) 81-94.

[35] R. Leary, Z. Saghi, P.A. Midgley, D.J. Holland, Compressed sensing electron tomography, Ultramicroscopy 131 (2013) 70-91.

[36] D.L. Chen, H. Friedrich, G. de With, On resolution in electron tomography of beam sensitive materials, J. Phys. Chem. C 118 (2014) 1248-1257.

[37] T.M. Peters, Algorithms for fast back and re-projection in computedtomography, IEEE Trans. Nucl. Sci. 28 (1981) 3641-3647.

[38] S.C.B. Lo, Strip and line path-integrals with a square pixel matrix - a unified theory for computational ct projections, IEEE Trans. Med. Imag. 7 (1988) 355-363.

[39] F. Xu, K. Mueller, A comparative study of popular interpolation and integration methods for use in computed tomography, in: proceedings of the 3rd IEEE International Symposium on Biomedical Imaging: Macro to Nano, Vols 1-3, 2006, pp. 1252-1255.

[40] K.J. Batenburg, J. Sijbers, Dart: A practical reconstruction algorithm for discrete tomography, IEEE Trans. Image Process. 20 (2011) 2542-2553.

[41] X. Yang, J. Loos, Toward high-performance polymer solar cells: the importance of morphology control, Macromolecules 40 (2007) 1353-1362.

[42] E.E. Tkalya, M. Ghislandi, G. de With, C.E. Koning, The use of surfactants for dispersing carbon nanotubes and graphene to make conductive nanocomposites, Curr. Opin. Colloid Interface Sci. 17 (2012) 225-232. 
[43] K.B. Lu, E. Sourty, R. Guerra, G. Bar, J. Loos, Critical comparison of volume data obtained by different electron tomography techniques, Macromolecules 43 (2010) 1444-1448.

[44] E.J. Kirkland, Advanced computing in electron microscopy, Springer, New York, 2010.

[45] M. Vulovic, R.B.G. Ravelli, L.J. van Vliet, A.J. Koster, I. Lazic, U. Lucken, H. Rullgard, O. Oktem, B. Rieger, Image formation modeling in cryo-electron microscopy, J. Struct. Biol. 183 (2013) 19-32.

[46] P.W. Hawkes, The electron microscope as a structure projector, Electron tomography: Threedimensional imaging with the transmission electron microscope, Plenum Press, New York (2006) 83-112.

[47] L. Reimer, H. Kohl, Transmission electron microscopy: Physics of image formation, 5th ed., Springer, New York, NY, 2008.

[48] W.J. Deruijter, Imaging properties and applications of slow-scan charge-coupleddevice cameras suitable for electron-microscopy, Micron 26 (1995) 247-275.

[49] J.M. Zuo, Electron detection characteristics of a slow-scan ccd camera, imaging plates and film, and electron image restoration, Microsc. Res. Tech. 49 (2000) 245-268.

[50] A.R. Faruqi, G. McMullan, Electronic detectors for electron microscopy, Q. Rev. Biophys, 44 (2011) 357-390.

[51] W.O. Saxton, W. Baumeister, M. Hahn, 3-dimensional reconstruction of imperfect two-dimensional crystals, Ultramicroscopy 13 (1984) 57-70.

[52] J.C. Russ, The image processing handbook, CRC press, 2010.

[53] R. Crowther, D. DeRosier, A. Klug, The reconstruction of a three-dimensional structure from projections and its application to electron microscopy, Proc. R. Soc. Lond. A 317 (1970) 319-340.
[54] W. Ming, M. Tian, R.D. van de Grampel, F. Melis, X. Jia, J. Loos, R. van der Linde Low surface energy polymeric films from solventless liquid oligoesters and partially fluorinated isocyanates, Macromolecules 35 (2002) 6920-6929.

[55] N. Grossiord, O. Regev, J. Loos, J. Meuldijk, C.E. Koning, Time-dependent study of the exfoliation process of carbon nanotubes in aqueous dispersions by using uv-visible spectroscopy, Anal. Chem. 77 (2005) 5135-5139.

[56] H. Heidari Mezerji, W. Van den Broek, S. Bals, A practical method to determine the effective resolution in incoherent experimental electron tomography, Ultramicroscopy 111 (2011) 330-336.

[57] A.J. Koster, M. Bárcena, Cryotomography: low-dose automated tomography of frozen-hydrated specimens, Electron Tomogr. (2006) 113-161.

[58] M. Radermacher, 3-dimensional reconstruction of single particles from random and nonrandom tilt series, J. Electron Microsc. Tech. 9 (1988) 359-394.

[59] N. Balakrishnan, Handbook of the logistic distribution, Dekker, 1992.

[60] M.C. Scott, C.C. Chen, M. Mecklenburg, C. Zhu, R. Xu, P. Ercius, U. Dahmen, B. C. Regan, J.W. Miao, Electron tomography at 2.4-angstrom resolution, Nature 483 (2012) 444-U491.

[61] A. Lange, A. Kupsch, M.P. Hentschel, I. Manke, N. Kardjilov, T. Arlt R. Grothausmann, Reconstruction of limited computed tomography data of fuel cell components using direct iterative reconstruction of computed tomography trajectories, J. Power Sources 196 (2011) 5293-5298.

[62] D. Wolf, A. Lubk, H. Lichte, Weighted simultaneous iterative reconstruction technique for single-axis tomography, Ultramicroscopy 136 (2014) 15-25. 